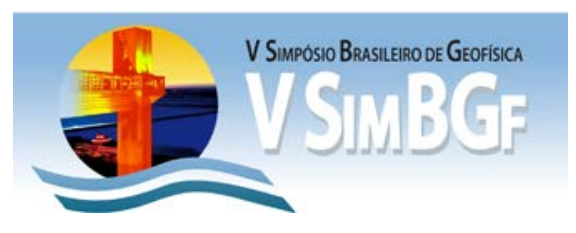

\title{
Determinação de padrões isotópicos de água usando espectroscopia de cavidade ressonante tipo ring down (ECRrd)
}

\author{
Tárcio Henrique Ribeiro dos Santos ${ }^{1}$; Maria do Rosário Zucchi ${ }^{1}$; Thierry Jacques Lemaire ${ }^{\star 1}$; Alexandre de Andrade Ferreira ${ }^{2}$; \\ Antônio Expedito Gomes de Azevedo ${ }^{1}$ \\ ${ }^{1}$ CPGG/IF-Universidade Federal da Bahia (UFBA) \\ ${ }^{2}$ CENPES-PETROBRAS
}

Copyright 2012, SBGf - Sociedade Brasileira de Geofísica

Este texto foi preparado para a apresentação no V Simpósio Brasileiro de Geofísica, Salvador, 27a 29 de novembro de 2012. Seu conteúdo foi revisado pelo Comitê Técnico do VSimBGf, mas não necessariamente representa a opinião da SBGf ou de seus associados. É proibida a reprodução total ou parcial deste material para propósitos comerciais sem prévia autorização da SBGf.

\begin{abstract}
In this paper we present the $\delta \mathrm{D}$ and $\delta^{18} \mathrm{O}$ results of one primary and two secondary standards commonly used for the determination of the isotopic composition of waters using the Cavity Ring Down Spectroscopy (CRDS) technique. These secondary standards were chosen to cover the range of values normally found in natural samples. One of them has both hydrogen and oxygen isotopic compositions close to those of sea water, while the other has relatively more depleted values, corresponding to water from the Alpes. The methodology, a simple one, does not require a lot of physical space in the laboratory and involves less time for analysis compared to conventional methods. Calibration was performed against the international standards VSMOW, GISP and SLAP, and presented excellent accuracy and precision, as given by standard deviations, $1.0 \%$ for $\delta \mathrm{D}$ and $0.18 \%$ for $\delta^{18} \mathrm{O}$.
\end{abstract}

\section{Introdução}

Atualmente, uma nova técnica de determinação de abundâncias de isótopos estáveis vem sendo empregada em larga escala. Trata-se da Espectroscopia de Cavidade Ressonante tipo ring down (ECRrd) (do inglês Cavity Ring-Down Spectroscopy, CRDS), uma técnica de absorção a laser que tem um grande potencial para a detecção de espécies atômicas e moleculares com uma alta sensibilidade. O ECRrd foi primeiramente introduzido por O'Keefe e Deacon em 1988. Consiste em uma técnica de absorção direta de alta sensibilidade e que se baseia na medida da razão de absorção da luz confinada dentro de uma cavidade óptica de alta finesse (capacidade da cavidade em manter um determinado grau de interferência construtiva), em vez da magnitude de absorção.

Este trabalho teve como objetivo determinar os valores da composição isotópica de duas águas que serão utilizadas como padrões secundários de referência do Laboratório de Física Nuclear Aplicada (LFNA-UFBA), denominados PB3 e PB4 e também um material certificado GISP, usado como verificador da qualidade dessas medidas. Para essa calibração, utilizou-se a escala isotópica SMOW-SLAP da Agência Internacional de Energia Atômica (AIEA) e a própria técnica ECRrd.

\section{Metodologia/ Problema Investigado}

As medidas das razões $\mathrm{D} / \mathrm{H}$ e ${ }^{18} \mathrm{O} /{ }^{16} \mathrm{O}$ em água são importantes, pois se aplicam a diversas áreas, tais como hidrologia, paleo-climatologia, química da atmosfera, geoquímica e biomedicina, no estudo metabólico de humanos e animais. Devido a esta grande variedade de aplicações e consequentemente variados tipos de amostras, é necessário que a análise das variações isotópicas apresente alta precisão, bem como exatidão e reprodutibilidade.

A maioria das moléculas compostas por $\mathrm{N}$ átomos apresenta $3 \mathrm{~N}-6$ graus de liberdade vibracional, exceto para moléculas lineares que tem $3 \mathrm{~N}-5$ graus de liberdade. Os núcleos vibram em torno de uma distância de equilíbrio e esse movimento vibracional é quantizado, de modo que a energia vibracional $E_{v}$, é:

$$
E_{v}=\hbar \omega\left(v+\frac{1}{2}\right), \quad \text { com } \quad \omega=\frac{1}{2 \pi} \sqrt{\frac{K}{\mu}}
$$

onde $v$ é o número quântico vibracional, $\mathrm{K}$ é constante elástica e $\hbar$ é constante de Planck normalizada. Pela análise dos espectros e estados vibracionais podemos determinar a abundância relativa dos isótopos, pois a frequência de vibração $\omega$, depende das massas dos átomos na molécula através da massa reduzida $\mu$. Então, se numa determinada amostra temos duas espécies isotópicas, as frequências vibracionais e os níveis de energia resultantes serão ligeiramente diferentes para os dois tipos de moléculas. Suas linhas espectrais, consequentemente estarão deslocadas umas em relação às outras $e_{2}$ pela medida das intensidades espectrais, podemos obter a abundância relativa dos isótopos.

$\mathrm{Na}$ ECRrd, o decaimento do pulso de luz é monitorado por um detector que mede a intensidade da luz transmitida através de um dos espelhos. Este decaimento é expressado com uma simples exponencial devido à transmissão através dos espelhos, dispersão e absorção molecular. A atenuação da luz viajando através da amostra é medida, e a intensidade transmitida através da cavidade (sendo $\mathrm{I}_{0}^{\prime}$ a intensidade inicial dentro da 
cavidade logo depois o primeiro espelho) segue a lei de Beer-Lambert.

$$
I_{0}=I_{0}^{\prime}(1-R) \exp (-\alpha L)
$$

onde $\mathrm{L}$ é o comprimento da cavidade em que está inserida a amostra caracterizada por um coeficiente de absorção $\alpha$. A intensidade medida pelo sensor decai exponencialmente no tempo com uma razão:

$$
\mathrm{k}=(1-\mathrm{R}) \frac{\mathrm{C}}{\mathrm{L}}
$$

onde c é a velocidade da luz e $\mathrm{R}$ é refletância dos espelhos. Define-se $1 / k=\tau$ como sendo o tempo de decaimento ring-down que corresponde ao tempo médio no qual, os fótons irão sobreviver dentro da cavidade. Portanto a distância efetiva que a luz percorre dentro da cavidade é

$$
\mathrm{L}_{\text {ef }}=\mathrm{C} \tau=\frac{\mathrm{L}}{1-\mathrm{R}}
$$

Basicamente, um equipamento com base na ECRrd é formado por dois espelhos de alta refletividade, acima de 99,99\%. Portanto uma pequena fração da luz será transmitida através de um dos espelhos para o detector a cada ciclo completo.

A intensidade dos pulsos decresce devido à absorção por espécies dentro da cavidade e reflexões nos espelhos. Assim, a intensidade do pulso no detector depois de $n$ ciclos completos é dada por:

$$
I_{n}=I_{0} R^{2 n} \exp (-2 n \alpha L)
$$

O tempo de cada ciclo completo (ida e volta dentro da cavidade) do pulso é $\mathrm{t}=\frac{2 \mathrm{~L}}{\mathrm{c}}$ e o tempo correspondendo a $\mathrm{n}$ idas e voltas na cavidade $\mathrm{t}_{\mathrm{n}}=\mathrm{n} \frac{2 \mathrm{~L}}{\mathrm{c}}$.

O termo $R^{2 n}$ pode ser escrito como $e^{2 n \ln R}$ e a intensidade após $\mathrm{n}$ ciclos fica

$$
\mathrm{I}_{\mathrm{n}}=\mathrm{I}_{0} \mathrm{e}^{2 \mathrm{n} \ln \mathrm{R}} \mathrm{e}^{-2 \mathrm{n} \alpha \mathrm{L}} \rightarrow \mathrm{I}_{\mathrm{n}}=\mathrm{I}_{0} \mathrm{e}^{2 \mathrm{n}(\mathrm{InR}-\alpha \mathrm{L})} \rightarrow \mathrm{I}_{\mathrm{n}}=\mathrm{I}_{0} \mathrm{e}^{\frac{\mathrm{ct}}{\mathrm{L}}(\ln \mathrm{R}-\alpha \mathrm{L})}
$$

Como os espelhos utilizados em ECRrd apresentam reflectância próxima de 1, podemos escrever $\ln R \cong-(1-R)$, e assim,

$$
I_{n}=I_{0} e^{\frac{c t}{L}(-1+R-\alpha L)} \rightarrow I_{n}=I_{0} e^{-\frac{c t}{L}(1-R+\alpha L)}
$$

O termo de decaimento que corresponde ao valor 1/e da intensidade inicial (do primeiro pulso) é justamente a constante de tempo ring-down $\tau$ definida por

$$
\tau=\frac{\mathrm{L}}{\mathrm{c}(1-\mathrm{R}+\alpha)}
$$

A rigor, deve-se levar em conta a extinção devido ao espalhamento da luz e sua absorção pelas diversas espécies presentes na cavidade. Todas estas contribuições são representadas pelo coeficiente de absorção, dependendo da freqüência: $\alpha(v)$. Portanto uma relação mais geral para o tempo de ring-down é dado por:

$$
\tau=\frac{L}{c\left[1-R(u)+\sum_{i} \sigma_{i}(v) \int_{0}^{L} N_{i}(x) d x\right]}
$$

onde a soma é feita sobre todas as espécies i com absorção dependentes da frequências ou secção de choque e a densidade do número de linha integrada. Observamos que as contribuições da extinção devido ao espalhamento da luz podem ser desprezíveis pelo fato da pressão do gás dentro da cavidade ser baixa.

Em ECRrd, o tempo de ring-down é medido como uma função da frequência do laser. Frequentemente o tempo de ring-down é obtido ajustando-se os dados da intensidade transitória da luz pelo método de mínimos quadrados. Um espectro de absorção é obtido plotandose a taxa de decaimento da cavidade ou as perdas da cavidade como função da frequência.

$$
\frac{1}{\mathrm{C} \tau(v)}=\frac{1-\mathrm{R}}{\mathrm{L}}+\alpha(v)
$$

onde o primeiro termo do segundo membro da equação representa a atenuação devido às reflexões nos espelhos determinada pela linha de base do espectro ECRrd e o segundo termo a perda por absorção da amostra.

O termo de absorção e consequentemente o coeficiente de absorção é obtido subtraindo-se as perdas em uma cavidade com amostra pelas perdas em uma cavidade sem amostra $\alpha=0$.

$$
\alpha(v)=\frac{1}{\mathrm{C} \tau(v)}-\frac{1}{\mathrm{C} \tau_{0}(v)}=\frac{\tau_{0}-\tau}{\mathrm{C} \tau_{0} \tau}
$$

Tal relação mostra que a absorção é determinada pela medida de duas taxas de decaimento ou dois tempos de ring-down (Figura 1). A amostra preenche toda a cavidade de modo que o coeficiente de absorção pode ser calculado sem conhecer o comprimento da amostra. Assim temos que:

$$
\alpha(v)=\frac{\tau_{0}-\tau}{\mathrm{c} \tau_{0} \tau}
$$

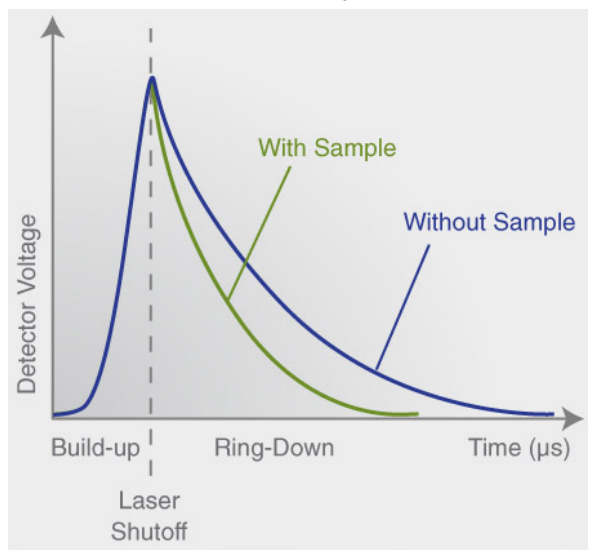

Figura 1. Tempo de "Ring Down" (fonte: WWW.PICARRO.com) 
O arranjo do ECRrd permite medidas de absorção sobre comprimentos de caminho óptico superiores a centenas de vezes o comprimento da cavidade, com o pulso podendo ser armazenado durante microssegundos fazendo centenas de idas e voltas.

\section{Procedimento analítico}

O aparato experimental para a ECRrd consiste em um sistema da marca PICARRO, contendo um laser I.V., uma cavidade ressonante tipo ring down formada por três espelhos altamente reflexivos, um detector (fotomultiplicador) e eletrônica digital para gravar os transientes de decaimento e um PC para o tratamento de dados (ver Figura 2).

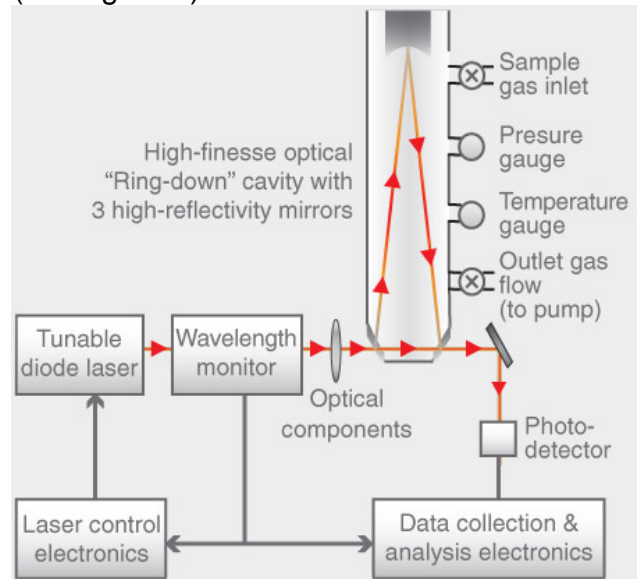

Figura 2. Diagrama esquemático (fonte: WWW.PICARRO.com)

Alíquotas de $2 \mu \mathrm{L}$ de água são injetadas em um vaporizador a $110{ }^{\circ} \mathrm{C}$. Posteriormente, o vapor é enviado ao analisador juntamente com $\mathrm{N}_{2}$, que é o gás de arraste. $\mathrm{Na}$ cavidade óptica, um sistema de medida baseado no tempo (ring-down) usa um laser para quantificar a característica espectral das moléculas na fase gasosa, especificamente as linhas de absorção únicas para $\mathrm{H}_{2}{ }^{16} \mathrm{O}, \mathrm{H}_{2}{ }^{18} \mathrm{O}$ e $\mathrm{HD}^{16} \mathrm{O}$ (Figura 3).

Com a medida baseada no tempo de decaimento da intensidade do feixe de luz, é possível determinar o coeficiente de absorção e consequentemente os valores isotópicos de hidrogênio e oxigênio, simultaneamente.

As variações na abundância isotópica dos isótopos estáveis encontradas em processos naturais são muito pequenas, por isso deve-se utilizar uma unidade de medida mais adequada. Assim, em 1950, McKinney introduziu uma notação especial que permite manipular mais facilmente as razões isotópicas. Introduziu a escala delta, $\delta$, que exprime, em \%o, por mil, o desvio das razões isotópicas relativamente a substâncias de referência ou padrão, de acordo com equação a seguir:

$$
\delta_{s / r}(\%)=\left[\frac{R_{s}}{R_{r}}-1\right] \times 10^{3}(\%)
$$

onde $R_{\mathrm{S}}$ é a razão isotópica entre o isótopo estável mais pesado e o isótopo estável mais leve na amostra e $R_{r}$ a razão isotópica entre o isótopo estável mais pesado e o isótopo estável mais leve na referência ou padrão.

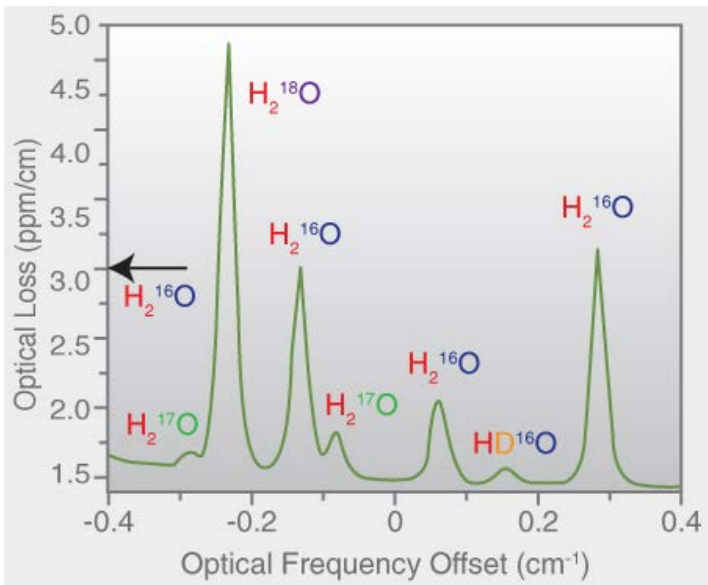

Figura 3. Espectro de absorção das espécies isotópicas da água. Absorção das diferentes espécies da molécula de água (fonte: www.PICARRO.com)

$\mathrm{Na}$ ECRrd, o valor $\delta$ pode ser calculado a partir da medida do coeficiente de absorção $\alpha_{n}$, onde $n$ refere-se ao isótopo mais leve ou mais abundante $\left(y=H^{16} \mathrm{OH}\right)$ ou mais pesado ou menos abundante $\left(x=\mathrm{H}^{18} \mathrm{OH}, \mathrm{H}^{17} \mathrm{OH}\right.$ ou $\left.\mathrm{H}^{16} \mathrm{OD}\right)$. Assim,

$$
\delta(x)=\frac{R_{x}^{\text {amostra }}}{R_{x}^{\text {padrão }}}-1=\frac{\left(\alpha_{x} / \alpha_{y}\right)^{\text {amostra }}}{\left(\alpha_{x} / \alpha_{y}\right)^{\text {padrão }}}-1=\frac{\left(\alpha_{x}^{\text {amostra }} / \alpha_{x}^{\text {padrão }}\right)}{\left(\alpha_{y}^{\text {amostra }} / \alpha_{y}^{\text {padrão }}\right)}-1
$$

\section{Resultados}

Os resultados de $\delta \mathrm{D}$ e $\delta^{18} \mathrm{O}$ dos padrões primários internacionais VSMOW, SLAP e GISP, da Agência Internacional de Energia Atômica (AIEA), e os padrões secundários PB3 e PB4 são apresentados nas tabelas abaixo, onde $n$ representa o número de medidas e $s$ o desvio padrão das medidas.

Tabela 1. Valores estabelecidos para o $\delta D$ e $\delta^{18} O$ e de desvio padrão (s) pela AIEA

\begin{tabular}{|c|c|c|c|c|c|c|}
\hline $\begin{array}{c}\text { PADRÕES } \\
(\mathrm{AIEA})\end{array}$ & $n$ & $\delta \mathrm{D}(\%)$ & $\mathrm{s}_{\delta \mathrm{D}}(\%)$ & $n$ & $\delta^{18} \mathrm{O}(\% \mathrm{o})$ & $\mathrm{s}_{\delta 180}(\%)$ \\
\hline VSMOW & & 0 & 0,3 & & 0 & 0,02 \\
\hline SLAP & & $-427,5$ & 0,3 & & $-55,5$ & 0,02 \\
\hline GISP & 75 & $-189,5$ & 1,2 & 83 & $-24,76$ & 0,09 \\
\hline
\end{tabular}

Tabela 2. Valores médios de $\delta D$ e $\delta^{18} O$ e de desvio padrão (s) das amostras de padrão estudadas.

\begin{tabular}{|c|c|c|c|c|c|c|}
\hline AMOSTRA & $n$ & $\delta \mathrm{D}(\%)$ & $\mathrm{S}_{\delta \mathrm{D}}(\% \mathrm{o})$ & $n$ & $\delta^{18} \mathrm{O}(\%)$ & $\mathrm{S}_{\delta 180}(\% \circ)$ \\
\hline GISP & 25 & $-188,9$ & 1,0 & 23 & $-24,75$ & 0,21 \\
\hline PB3 & 19 & $-2,3$ & 1,3 & 35 & $-2,13$ & 0,18 \\
\hline PB4 & 29 & $-71,5$ & 0,9 & 36 & $-10,05$ & 0,16 \\
\hline
\end{tabular}




\section{Discussão e Conclusões}

Neste trabalho, foram preparados dois padrões isotópicos secundários de água, PB3 e PB4. O primeiro refere-se a uma água mineral local, com valor isotópico próximo ao da água do mar, e o segundo uma água mineral originária dos Alpes, bastante empobrecida em isótopos pesados. A calibração desses padrões foi realizada em relação a escala isotópica VSMOW-SLAP (AIEA). Utilizou-se o padrão certificado GISP (AIEA), como controle de qualidade das medidas, cujos resultados estão apresentados na Tabela 2. Verificou-se uma excelente exatidão e boa precisão dada pelo desvio padrão de $1.0 \%$ para o $\delta \mathrm{D}$ e $0.18 \%$ para o $\delta^{18} \mathrm{O}$. Desta forma, pode-se concluir que os padrões secundários PB3 e PB4 foram calibrados com sucesso e que a técnica ECRrd apresenta uma alternativa promissora na determinação isotópica de hidrogênio e oxigênio de amostras de água.

\section{Agradecimentos}

PETROBRAS e a CAPES pelo apoio financeiro.

\section{Referências}

BRAND, W. A.; AVAK, H.; SEEDORF, R.; HOFMANN, D.; CONRADI, T. New methods for fully automated isotope ratio determination from hydrogen at the natural abundance level. Geoph. Prosp., 28:967-976. 2000.

BERDEN, G.; ENGELN, R. Cavity Ring-Down Spectroscopy - Techniques and Applications. United Kingdom: John Wiley \& Sons Ltd, 2009.

E.R.T. KERSTEL, R. VAN TRIGT, N. DAM, J. REUSS, H.A.J. MEIJER. Laser Spectrometry Spplied to the Simultaneous Determination of the $2 \mathrm{~h}, 17 \mathrm{o}$, and 180 Isotope Abundances in Water. Centrum voor Isotopenonderzoek, University of Groningen, Groningen, Netherlands. New Approaches for Stable Isotope Ratio Measurements, IAEA, 1999.

NAKAEMA, W. M. Espectroscopia de cavidade ressonante tipo Ring-Down Supercontinuum resolvida no tempo para detecção de multicomponentes gasosos. Tese de doutorado, Instituto de Pesquisas Energéticas e Nucleares IPEN, São Paulo, 2010.

O'KEEFE, A.; DEACON, D.A.G. Cavity ring-down optical spectrometer for absorption measurements using pulsed laser sources. Review of Scientific Instruments, V.59, P. 2544-2551, 1988.

ZALICKI, P.; ZARE, R.N. Cavity ring-down spectroscopy for quantitative absorption measurements. J. Chem. Phys. V. 102, p. 2708-2717, 1995.

PICARRO: www.picarro.com.br. 